\title{
Philosophiques
}

\section{Réconciliation et dépassement de l'art par la philosophie chez Hegel : une analyse critique}

\section{Syliane Charles}

Volume 25, numéro 1, printemps 1998

URI : https://id.erudit.org/iderudit/027471ar

DOI : https://doi.org/10.7202/027471ar

Aller au sommaire du numéro

\section{Éditeur(s)}

Société de philosophie du Québec

\section{ISSN}

0316-2923 (imprimé)

1492-1391 (numérique)

Découvrir la revue

\section{Citer cet article}

Charles, S. (1998). Réconciliation et dépassement de l'art par la philosophie chez Hegel : une analyse critique. Philosophiques, 25(1), 49-61.

https://doi.org/10.7202/027471ar

\section{Résumé de l'article}

Hegel attribue à l'art et à Ia philosophie une même tâche dans l'histoire de l'Esprit, celle de "réconcilier » les opposés. Nous expliquerons de quels éléments à médiatiser il s'agit, et une étude détaillée de textes de l'Introduction à l'Esthétique nous amènera à critiquer le moment de la transition vers la philosophie, celui de la poésie romantique. Nous suggérerons en particulier que l'emploi de deux critères différents pour établir la hiérarchie des arts et des formes empêche Hegel de justifier réellement le dépassement de l'art par la philosophie ainsi que la supériorité de la réconciliation philosophique. 


\title{
RÉCONCILIATION ET dÉ PASSEMENT DE L'ART PAR IA PHIIOSOPHIE CHEZ HEGEL : UNE ANALYSE CRIIIQUE*
}

\author{
PAR \\ SYIIANE ChaRLES
}

\begin{abstract}
RÉSUMÉ : Hegel attribue à l'art et à la philosophie une même tâche dans lhistoire de l'Esprit, celle de "réconcilier "les opposés. Nous expliquerons de quels éléments à médiatiser il s'agit, et une étude détaillée de textes de / Introduction à l'Esthétique nous amènera à critiquer le moment de la transition vers la philosophie, celui de la poésie romantique. Nous suggérerons en particulier que l'emploi de deux critères différents pour établir la hiérarchie des arts et des formes empêche Hegel de justifier réellement le dépassement de l'art par la philosophie ainsi que la supériorité de la réconciliation philosophique.
\end{abstract}

ABSTRACT: Hegel assigns to art and philosophy one and the same task in the history of the Spirit, that of "reconciling" the opposites. I explain which elements are mediated, and rely on a detailed study of extracts from the Introduction to Aesthetics to criticize the moment of the transition to philosophy, viz. romantic poetry. In particular, I suggest that the use of two different criteria to establish a hierarchy of arts and forms prevents Hegel from giving a proper justification of how philosophy transcends art, and of why its reconciliation is superior.

Dans les cours donnés à Berlin sur l'esthétique de 1820 à 1829 , Hegel entend montrer dans quelle mesure l'art fait partie de l'histoire de réalisation de l'Esprit, ou du Concept (der Begriff), c'est-à-dire dans quelle mesure il est un moment de ce qu'il appelle la prise de conscience de soi ou encore la détermination de l'Esprit. La tâche qu'il attribue à l'art, dans cette histoire idéale et idéelle, est d'opérer la réconciliation des deux pôles contraires que sont le spirituel et le naturel, ce qui se comprend bien par la forme même de ce médium qui réunit les deux éléments. Mais à ses yeux, la forme sensible finit par se trouver insuffisante à contenir l'Esprit lorsque celui-ci parvient à une détermination plus haute, de sorte qu'il s'incarne d'une

- Une première version de ce texte a fait l'objet d'une communication lors des séances de la Société de philosophie du Québec au congrès 1997 de l'ACFAS à l'Université du Québec à Trois-Rivières. 
manière privilégiée dans la religion, puis enfin dans la philosophie. Celle-ci, d'après Hegel, réalise parfaitement la « réconciliation " dont il parle, et marque ainsi le parachèvement de la réalisation du Concept. Notre objectif ici est moins de soutenir une thèse que de chercher à penser avec Hegel, et à faire ressortir les implications de sa pensée, dans la mesure où nous la comprenons. Pour ce faire, nous entendons clarifier le sens des notions de réconciliation et de médiation (Versöhnung/Vermittlung) et mettre en lumière leur importance. Ce faisant, nous nous demanderons dans quelle mesure le système hégélien justifie le dépassement de l'art par la philosophie ainsi que l'insuffisance de la réconciliation que celui-ci opère par rapport à la réconciliation philosophique. Notre questionnement, à partir d'un examen de la lettre du texte de Hegel, devra alors se muer en analyse criticfue.

\section{La réconciliation dans l'art et dans la philosophie}

La philosophie de Hegel est fondée sur une conception à deux niveaux de la réalité. La " nature "est l'opposé de l' Esprit », son altérité radicale, et ces deux forces se retrouvent face à face dlans tous les aspects de la vie et de l'histoire humaine. Se définissant l'un par l'autre (l'esprit est le non-naturel, la nature est ce qui est dénué (l'esprit), ces (leux éléments entrent en conflit dans leur constitution bilatérale du monde, car l'être humain vise à rendre l'extérieur, dans lequel il évolue. compréhensible et maîtrisable. La "nature "sc comprend donc dans un premier moment comme ce qui est " donné „à l'état brut, et non encore spiritualisé par l'homme. Par conséquent, comme Hegel l'affirme au quatrième paragraphe de l'Introduction à l'Esthétique, la supériorité à la fois ontologique et axiologique de l'Esprit sur la nature implique la supériorité des produits de la pensée sur les produits naturels, ce qui implique qu'une réalisation a d'autant plus de réalité et de valeur qu'elle est abstraite, qu'elle a peu de rapports avec le sensible qui est " l'Autre orle la pensée.

Doù. semble-t-il, à la fois la valeur cle laart, qui est plus que la simple nature, car il est essentiellement ceuvre humaine, et son infériorité vis-à-vis de la religion et de la philosophie. cette dernière en particulier représentant la forme d'expression la plus haute de l'Esprit, la plus épurée de tout élément sensible. Dans l'art, il est question d'associer adéquatement un contenu idéal (tel que, par exemple, un concept, le divin, la liberté...) à une forme sensible. une matérialité qu'il s'agit pour l'artiste de modeler de façon à ce que le sens soit le mieux possible "exprimé » ou « incarné " par ce qui le donne à voir ou à entendre. L'œuvre d'art est le lieu d'une r'éconciliation par le fait que chaque cuvre comporte les deux versants, idéal et sensible : "Dans le même sens, il nous faut dire de l'art qu'il vise à transformer chaque forme, en tous les points de la 
surface sensible, en œil, celui-ci étant le siège de l’âme et le lieu de manifestation de l'esprit ${ }^{1}$ m.

Mais l'art n'est qu'un premier stade d'expression de l'Esprit dans le monde, stade où la forme sensible reste le seul moyen d'accéder à la spiritualité du contenu : “ Ce n'est que dans sa liberté que le bel arı est lart véritable, et celui-ci ne parvient à réaliser sa tâche la plus haute que quand il s'est placé dans la sphère qui hui csl communc avec la religion et la philosophie, et qu'il n'est plus qu'une manière d'exprimer et de faire prendre conscience du divin, des intérêts les plus profonds de l'homme, des vérités totales de l'esprit ${ }^{2}$ ». L'Esprit qui s'est déterminé trouve son expression la plus adéquate dans la forme divine, et c'est le moment religieux, avant que l'homme luimême ait conscience que c'est dans sa raison que s'incarne lc Concept et que son expression véridique est dans la pensée, c’est-àdire dans la philosophie. L’art se trouve donc dépassé par ces deux autres moyens d'expression de l'Esprit, et la philosophie est le plus parfait de tous. Or, c'est à ce niveau que la tâche est à la fois réalisée, et toujours à réaliser, comme le dit Hegel dans son Introduction ì l'Esthétique: “Cette médiation n'est pas une exigence vide, mais ce qui est réalisé en soi et pour soi et qui se réalise éternellement n « das an und für sich Vollbrachte und stets sich Vollbringende ${ }^{3}$ ), car c'es la double nature de l'homme qui fait obstacle à sa spiritualisation totale, et c'est de la réconciliation dans la forme humainc elle-même que s'occupe la philosophié.

L'opposition fondamentale de l'Esprit et de son Autre, du subjectif et de l'objectif, prend en effet plusieur's formes dans lesquelles elle ne peut se résoudre clle-même complètement, dans lesquelles, en quelque sorte, la dialectique narrive pas à "absorber" », à dépasser la contradiction. Le conflit des deux éléments se retrouve, sur le plan de la morale humaine, entre liberté et nécessité, entre loi morale universelle et flux du particulier, entre conscience réfléchice a passions ou sentiments et dans ce que Hegel va jusqu'à appeler a la lutte du spirituel contre la chair » (" der Kampf des Geistiges gegen das"

1. G. W. Hegel, Werke, Band 13 : Vorlesungen über die Ästhetik, Suhrkamp Verlag, Frankfurt am Main, 1970, I, III, A, p. 203 : "In demselben Sinne ist von der Kunst z." behaupten, daßs sie jede Gestalt an allen Punkten der sichtbaren Oberfläche zum Auge verwandle, welches der Sitz der. Seele ist und den Geist zur Erscheimung bringt n. Les traductions, que nous avons voulues aussi littérales que possible - d'où, parfois, leur inélégance - , sont nôtres.

2. "In dieser ihrer Freiheit nun ist die schöne Kunst erst svahrhafte Kunst und löst dann erst ihre höchste Aufgabe, wenn sie sich in den Geneinschaftlichen Kreis mit der Religion und Philosophie gestellt hat und nur eine Art und Weise ist, das Gottliche, die tiefsten Interessen des Menschen, die umfassenden Wahrheiten des Geistes zum Bewußtsein zu bringen und auszusprechen. "Ibid., Einleitung I, p. 20-21

3. Ibid., Einleitung III, 3, p. 82.

4. Hegel emploie bien le terme a philosophie "dans son Introduction à l'Esthétique, mais en parallèle avec celui de " science » qu'il avait utilisé de manière privilégiée auparavant. 
Fleisch ${ }^{5}$ m. Or, d'après Hegel, c'est la culture moderne qui a aggravé cette tension en montrant qu'elle se trouve au sein même de l'homme, de sorte qu'il est devenu comme un être " amphibie ", selon ses propres termes : "L'éducation spirituelle, l'entendement moderne portent cette contradiction au plus haut point en l'homme, et celle-ci en fait un être amphibie, car il doit désormais vivre dans deux mondes qui se contredisent ${ }^{6}$ ». Ne parvenant pas à faire triompher en lui ou le pôle des passions, ou celui de la raison, l'homme moderne est tombé dans une instabilité qui lui cause une insupportable inquiétude. Ni l'Esprit pur ni le sensible brut ne peuvent être à eux sculs la vérité, car l'homme est composé des deux et l'on ne peut pas imaginer que l'un puisse jamais anéantir l'autre totalement. Si on les prend séparément, chacun des termes opposés perd d'ailleurs son sens, puisque Esprit et nature ne se comprennent que négativement l'un par rapport à l'autre, comme étant tout ce que l'autre n'est pas. Hegel en conclut que la vérité pour l'homme ne peut résider que dans la conciliation des deux et que l'Esprit doit quitrer son abstraction pour investir concrètement le vécu humain, sans quoi l'on reste dans une situation tragique. Or, l'art opère une première médiation entre les deux opposés pris alors comme fond et forme, mais Hegel vise à montrer que celle-ci est inférieure à la réconciliation conceptuclle qui est le fait de la philosophie.

La pensée plonge dans la profondeur d'un monde suprasensible, qu'elle pose ensuite comme un arr-delà de la conscience immédiate et de la sensation actuclle; la liberlé de la connaissance réflexive se clélivre de l'en-deçà représenté par la réalité sensible et la finitude. Mais cette rupture, à laquelle parvient l'esprit, il sait également la soigner ; il engendre à partir de lui-mêne les ceuvres clu bel art qui constituent le premier moyen terme entre ce qui est simplement extérieur, sensible et éphémère, et la pensée pure ; entre la nature et la réalité finie, et la liberté infinie de la pensée conceptuelle?

Comme la fin de ce passage le montre bien, Hegel considère que l'art est un premier pas, et non le dernier, dans l'entreprise de réconciliation entre le spirituel et le naturel - et sachant que la philosophie vise aussi à réconcilier ces deux instances opposées, il

5. Ibid. p. 80 .

6. Ibid., p. 80-81 : "Die geistige Bildung, der moderne Verstand bringt im Menschen diesen Ciegensazz hervor. der ihn zur Amphibie macht, indem er num in zwei IVehen zul leben hat, die sich swidersprechen ".

7. "Es ist die Tiefe ciner übersimnlichen Welt, in welche der Gedanke dringt und sie zunächst als ein Jenseits dem unmittelbaren Berwußtsein und der Gegenwärtigen Empfindung gegenüber aufstellt; es ist die Freiheit denkender Erkenntmis, we/che sich dem Diesseits, das simnliche Wirklichkeit und Endlichkeit heißt, enthebt. Diesen Bruch aber, zut welchem der Geist fortgeht, weiß er ebenso zu heilen; er erzeugt aus sich selbst die Werke der schönen Kunst als das erste versölnende Wittelglied zuischen dem blofs Außerlichen, Sinnlichen und Vergänglichen und dem reimen Gedanken, zwischen der Natur und endlichen Wirklichlieit und der unendlichen Freiheit des begreifenden Denkens n, ibid., p. 21. Comme S. Jankélévitch (clans Introduction à l'Esthétique, Paris, éd. Aubier-Montaigne, 1964, p. 41), nous avons modifié la construction de la première phrase pour la traduire. 
apparaît clairement que Hegel lui assigne le même but qu'à l'art. Si l'on se fie à l'exposé qui en est donné dans les Leçons sur la philosophie de l'histoire, on peut dire que chez Hegel l'Esprit passe par quatrc grandes époques historiques dans son développement et sa prise de conscience de lui-même : 1) il prend naissance dans le monde oriental de manière indistincte vis-à-vis de la nature, qui esi divinisée ; 2) il revêt dans la civilisation grecque sa première forme humaine, mais reste entaché de trop de particularismes sensibles ; 3) il acquiert son abstraction pleine dans le monde romain, mais est alors placé comme une nécessité transcendante à l'homme; enfin 1) l'homme est réconcilié avec lui-même dans le monde germanique moderne et le moment de la philosophie, car l'Esprit rejoint l'esprit réfléchissant, il se saisit à la fois dans sa forme humaine et dans son universalité. Comment interpréter l'idée que la philosophie parvient à cette réconciliation? Penchons-nous un peu plus longuement sur un passage troublant, et a fortiori très ćclairant, de l'Introduction à l'Esthétique de Hegel :

Si la culture générale est tombée clans une telle contracliction, alors il incombe à la philosophie de résondre les oppositions, c'est-à-dire de montrer dic Gegensärze anfzuheben ${ }^{8}, d$. i. zu zeigenj que ni l'un, pris dans son abstraction, ni l'autre, clans la même unilatéralité, n'a la vérité, mais qu'ils sont tous les deux ce qui se clissout soi-mêne (das Sichselbstauflösende), et que la vérité ne réside que dans la réconciliation et clans la médiation cles deux?

Il semble que le rôle de la philosophie soit double, mais que ces deux rôles soient en réalité chacun une face de l'autre, comme l“indique le « das heißt», le "c'est-à-dire », qui les met sur un plan d"égalité. On trouve tout d'abord le rôle traditionnel de la philosophie dans la dialectique hégélienne, qui consiste à résoudre les oppositions (die Gegensätze aufheben). et le second, cclui d'un simple instrument pour comprendre et indi(fuer (zeigen) comment se déroule ce processus. Il ny a pas contradiction si lion comprend que ce processus de résorption du dualisme entre Esprit et nature n'est pas purement extérieur à la philosophie, mais qu'elle seule peut comprendre son propre concept et sa propre essence. De sorte que par l'esprit avec une minuscule, les hommes peuvent refaire, grâce à la philosophic (et Hegel pense en particulier à la sienne), le chemin suivi par l'Esprit absolu dans sa médiation avec son Autre. C'est un cercle : l'Esprit, depuis toujours, s'oppose à la nature dans les actions humaines, mais

8. Le verbe est précisément ici celui qui sert à former le mot " clialectique ", "die Aufhebung ".

9. Ibid., p. 81-82 (c'est nous qui soulignons) : "Ist die allgemeine Bildung in dergleichen Widerspruch hineingeraten, so wird es die Aufgabe der Philosophie, die Gegensätze aufzuheben, d. i. zu zeigen: weder der eine in seiner Abstraktion noch der andere in gleicher Einseitigkeit hätten Wahrheit, sondern seien das Sichselsbtauflösende; die Wahrheit liege erst in der Versöhnung und Vermittlung beider, und diese Vermittlung sei keine bloße Forderung, sondern das an und für sich selbst Vollbrachte und stets sich Vollbringende $\boldsymbol{}$. 
par leur réflexion et l'évolution de leur conscience morale, les hommes comprennent la supériorité de l'Esprit et contribuent à lui faire investir chacune de leurs actions, donc à imposer sa marque au monde naturel (en eux et à l'extérieur). La philosophie, parce qu'elle offre les moyens conceptuels pour comprendre et expliquer cette circularité dialectique, serait donc elle-même à la fois ce qui permet l'investigation par l'Esprit de l'ensemble du réel (d'où la résorption du conflit) et son instrument de compréhension pour les hommes.

Cette réconciliation "des deux esprits" dirions-nous presque, Esprit avec une majuscule et esprit avec une minuscule, ou pensée, c'est la réconciliation de l'universel et du particulier, qui est une des f'ormes de l'opposition originelle entre spirituel et naturel ; et elle marcfue bien le parachèvement de l'histoire de la détermination de l'Esprit. Il était done nécessaire philosophiquement que Hegel ménage un passage dialectique d'une sphère à l'autre jusqu'à celle de la philosophie, c'est bien là une chose acquise. Ce qui, en revanche, nous semble problématique, comme nous allons tenter de le faire voir, c'est l'explication apportée par le système hégélien lui-même' pour justifier la supériorité de la réconciliation philosophique vis-à-vis de celle que propose l'art. Précisément, en analysant les formes d'art où s'opère cette réconciliation, on voit que nous n'avons absolument pas besoin de faire appel à la notion d'homme, ce qui indique, nous semble-1-il, que ce n’est pas la même chose qu’il s’agit de réconcilier - la philosophie, nous l'avons vu, est avant toul réconciliation de l'Esprit absolu avec l'esprit particulier de l'homme. Voyons précisément comment IIegel décrit le passage, postulé comme "nécessaire " par la théorie, entre l'art et la philosophie, à travers le moment de la poésie. Si l'on n'y trouve pas la nécessité posée par la science de l'esprit de Hegel, nous aurons une indication du fait que l'art et la philosophie, s'ils médiatisent tous deux certes des contraires, ne visent peut-être pas la même réconciliation, de sorte que le passage d'une sphère à l'autre reste problématique.

\section{La poésie, “ art général de l'esprit » et « moment où l'art se dépasse lui-même »}

Liidée principale exposée par I Hegel aux pages 122 et 123 de son Inroduction, clans l'édition allemande, est que la poésie, au sens précis dans lequel ce terme était employé à son époque, c'est-à-dire la forme artistique regroupant les genres épique, lyrique et dramatique, représente la forme artistique la plus apte, dans l'histoire de la prise de conscience de soi du Concept, à exprimer un contenu spirituel concret, déterminé en soi et pour soi. Si la poésie est la forme la plus adaptée pour exprimer le spirituel dans sa plénitude, c’est parce qu'elle est la forme d'art la plus spirituelle. Par conséquent, écrit Hegel, "c’est précisément à ce degré le plus haut que l'art se transcende aussi lui-même, en abandonnant l'élément de la matérialisation réconciliée de l'esprit pour passer de la poésie de la 
représentation mentale à la prose de la pensée ${ }^{10}$ ". Or', d'oì tire-t-elle ce statut, pourquoi est-ce au moment de la poésie en particulier que l'art touche à sa fin? Par ailleurs, en quoi peut-on considérer que la poésie est privilégiée en spiritualité vis-à-vis des autres formes artistiques? Nous ne pouvons comprendre ce statut supérieur quien replaçant brièvement la poésie dans la suite hiérarchique des formes artistiques dans laquelle l'insère Hegel, ce qui nous amènera à unc discussion critique à partir de la reconstruction détailléc de son argument.

Hegel divise l'histoire de lart en trois grands moments qui correspondent chacun à un rapport diffécent entre le fond el la forme. Lart symbolique, qui sexprime essentiellement a lravers l'architecture, donne forme d'après Hegel à un contenu idéal indéterminé et vague. de sorte que la forme sensible est Iolalement insuffisante à exprimer adéquatement ce contenu qui la déborde de toutes parts et ce, non par une faiblesse de celle-ci, la forme artistique, mais par un manque de détermination de celui-là. l'Esprit. L'art classique, en revanche, correspond au point d'équilibre parfait cutre le fond et la forme : L'esprit, devenu transparent à soi-même, se signifie (porte en soi et sa signification el son interprétation). s'objective (revêt, en tant que son propre objer, une apparence qui ne peut le trahir puisqüil s"y reconnaît), bref, se rejoint. ll n'y a plus ui opposition, ni même distinction, entre forme et contenu" „. C"est là un point important, car nous voudrions insister sur le fait fue la réconciliation qui est la tâche de l'art semble déjà effectuéc parfaitement au niveau du classifue. Comme on le voil en particulicr clans l'art sculptural antique, le contenu est conçu sous une forme déterminéc, la forme humaine, et celle-ci est justement la plus adéquate à l'esprit. Cette concordance harmonieuse entre l’idéal et la forme sensible marque l'apogée de l'art sur le plan esthétique, les cuuvr's d’art classique devant done, en vertu du critère de l'adéquation du fond et de la forme, être les plus abelles "róalisations de l'esprit humain. Ce sont bien aussi celles qui réalisent le mieux la réconciliation entre l'élément sensible et l'élément spiritucl dans l'art.

Toutefois, le Concept qui, en tant que contenu idéal de l'aruvre d'art, s'est conçu comme humain dans la forme classique, continue d'après Hegel à prendre conscience de lui-même dans des objets de plus en plus élaborés spirituellement. Et plus il croît con détermination, moins la forme sensible peut le contenir, de sorte que l'on parvient avec l'art romantique (qui est le troisième grand moment de l'art) à un débordement de la forme artistique par le. contenu, celui-ci ćtant cette fois trop déterminé, nous dit Hegel, pour

10. Ibid., p. 123 : a Doch gerade auf deiser höchsten Stufe steigt nun die Kunst auch über sich selbst hinaus, indem sie das Element versöhnter Versinnlichung des Geistes ver/äßst und aus der Poesie der Vorstellung in die Prosa des Denkens übertritt $\mathrm{n}$.

11. Bernard Tesseydre, L'Esthétique de Hegel, Paris, PUF, 1963, p. 75. 
être pleinement exprimé dans le sensible. De plus, la forme d'art romantique nous est présentée par Hegel comme étant caractéristique de trois arts en particulier, à savoir la peinture, la musique et la poésie. Dans cet ordre, il établit une nouvelle hiérarchie entre eux en fonction de leur moindre degré de matérialité, le son étant plus éthéré (done plus spirituel) que les couleurs de la peinture et la poésie parachevant la subordination de la forme sensible au sens idéal, car en elle, "le son devient mot, en tant que son articulé en lui-même dont le sens est de dépeindre des représentations mentales et des pensées ${ }^{12}$ " et non plus de désigner des sentiments ou des passions, comme dans la musique. Ia question (fui est la nôtre ici est la suivante : comment Hegel justifie-t-il la nécessité de dépasser l'art classique?' Ce dépassement, on le sait, est opéré par l'art romantique, et en particulier par la poésie, qui en est la forme par excellence. C'est ce que l'on trouve exposé dans l'extrait ci-dessous ; mais nous verrons également que ce passage à une troisieme forme d'art se justifie par une compréhension biaisée, "métaphysique " et non plus a artistique ", de la poésie par Hegel. Voyons exactement ce qu'il en est, avant de revenir sur la question d'une possible contradiction entre un postulat spirituel et un postulat esthétique dans l'explication du développement de l'art :

Pour ce ru'il en est, enfin, de la troisième représentation, spirituelle, de la forme d'art romantieque, il nous faut la chercher clans la poésie. Sa particularité caractéristique résicle dlans le pouvoir qu'elle a d'assujettir à l'esprit et à ses représentations l'élément sensible, dont la musique et la peinture avaient rléjà commencé à lihérer l'art. Car le son, dernier matériau extérieur cle la poésie, n'est plus en elle un écho rle la sensation elle-même, mais un signe pour soi insignifiant. et, en vérité, celui de la représentation mentale devenue concrète en elle-même, el non pas seulement le signe de la sensation indéterminée avec ses nuances et ses gradlations [...]. Cet élément sensible [le son], qui dans la musique faisait encore immédiatement un avec l'intériorité, est ici [đlans la poésie] séparé du contenu de la conscience, tandis que l'Esprit détermine ce contemu, pour soi et en soi, en une représentation mentale, pour l'expression de laquelle, certes, il se sert du son, mais seulement conme d'un signe dépourvu pour soi de valeur' et de contenu. Par conséquent, le son peut tout aussi bien être une simple lettre de l'alphabet, car l'audible, tout comme le visible, se trouve ravalé au rang rle simple indice de l'Esprit. L'élément propre à l'exposition poétique est ainsi la représentation el l'illustration de l'Esprit, et comme cet élément est commun à toutes les formes d'art, la poésie les traverse toutes et se développe librement en elles. L'art poétique est la forme générale de l'Esprit devenu libre en lui-même, dont la réalisation n'est plus liée an matériau externe-sensible, et qui ne se déploie plus que dans l'espace interne et dans le temps interne des représentations mentales et des sensations. Er pourtant c'est précisément à ce clegré le plus haut que l'art se

12. "Der Ton wird dadurch zum Wort als in sich artikuliertem Laute, dessen Sinn es ist. Vorstellungen und Gedanken zu bezeichnen. "Werke, 13, Einleitung, p. 122. 
transcende aussi lui-même, en abandonnant l'élément de la matérialisation réconciliée de l'Esprit pour' passer de la poésie de la représentation mentale à la prose de la pensée $e^{13}$.

L’idée selon laquelle la poésie est la forme la plus spirituelle et la forme supérieure des arts est soutenue par l"affirmation, d"unc par. que dans la poésie l'élément sensible est assujetli à l'élément spirituel, à la différence des autres types d'ouvres el, d'autre part., par' le postulat que la spiritualité a plus de valeur que la sensation. De là, on peut dire que puisque la poésie est proprement le lieu d’incarnation de la spiritualité en art. et puisqu'elle est commune à toutes les formes d'art (symbolique, classique el romantique), clle est " l'art général de l'esprit", au sens quantitatif tout aulant que: qualitatif. On connaît l’admiration égale de Hegel pour la Iragédie antique (Antigone en particulier) et pour les poèmes lyriques de Goethe ou Hölderlin. La poésie est commune à toutes les époques, et bien que ce soit dans la forme de lart romantique qu'elle atteint. d'après Hegel, son plus haut degré de spiritualité ${ }^{11}$, elle reste dans chaque moment le moyen d'expression le plus adécuat du contenu idéal. Et ce, parce qu’elle utilise le langage qui. dans sa matérialité do signe, renvoie tout de suite à autre chose qu'à lui-même, renvoie à un sens, à une idée.

13. Ibid., p. 122-123 : Was endlich die dlitte, geistige Darstellung der romantischen Kunstform anberriff. so haben wir dieselbe in der Poesie zu suchen. Ihre charakteristische Eigentümlichkeir liegt in der Macht. mit welcher sie das sinnliche Element, von dem schon Musik und Malerei dic Krunst zu befreien begannen, dem (iciste und seinen Vorstellungen untersvirft. Denn der Ton, das letzte äußere Material der. Poesie, ist in ihr nicht mehr die tönende Enypfindung selber, sondern cin für sich beteutungsloses Zeichen, und zwar der in sich konkret gevordenen Vorstellumg, nich aber nur der unbestimmten Empfindung und ihrer Nurancen und Grodationch [...]. Doch ist dies simnliche Element /der Ton/, das in der Whsik noch unmittelbar eins mit des. Innerlichkieit war, hier /in der Poesie/ on dem Inhalte des Beswuftscins losgctremm. während der Geist diesen Inhalt sich für sich und in sich selbst zur Vorsellung bestimm! zu deren Ausdruck er sich zuar des Tones, doch mur als eines fiir sich west - und inhaltlosen Zeichens bedient. Der Ton kann demmach chensogut auch bloficr Buchstabe sein, denn das Hörbare ist swie das Sichtbare zur bloken Andeutung des Ceistes herabgesunken. Dadurch ist das eigenthiche Element poetischer Darstellung die poetische Vorstellung und geistige Veranschaulichung selber, und indem dies Element allen Kunstformen gemeinschaftich ist, so zieht sich anch die Poesie durch alle hindurch und entwickelt sich selbständig in ihnen. Die Dichthunst ust die allgemeine Kunst des in sich freigewordenen, nicht an das äßSerlich-sinnliche Material zur Realisation gebundenen Geistes, der nur im inneren Raume und der inneren Zeit der Vorstellungen und Empfindungen sich ergeht. Doch gerade auf dieser höchsten Stufe steigt mun die Kunst auch über sich selbst hinaus, indem sie das Element versöhnter Versinnlichung des Geistes verläßt und aus der Poesie der Vorstellung in die Prosa des Denkens himübertritt n.

14. C'est d'ailleurs, à nos yeux, le seul postulat qui justifie que Hegel puisse dire de la poésie qu'elle est la forme artistique générale de l'esprit, car en ce qui concerne le versant strictement quantitatif, elle se trouve au même niveau cue tous les autres types de réalisation artistique (l'architecture, par exemple, qui caractérise justement la forme la plus inférieure de l'art, c'est-à-dire le symbolique, est tout aussi présente dans tous les autres grands moments de l'art, à savoir classique et romantique, que l'est la poésie). 
Une autre conclusion est toutefois aussi possible à partir de l'affirmation que la poésie est la forme la plus spirituelle, si l'on ajoute comme deuxième prémisse l'idée présente dans toute l'cuuvre de Hegel que le Concept s'incarne dans toutes les formes de réalisations humaines. Puisque l'expression la plus parfaite de l'esprit est conceptuelle et non pas sensible, et que la poésie est la forme artistique la plus spirituelle, on parvient à l'idée que c'est dans la poésic que l'art se transcende lui-même. Le passage cité tient ces deux affirmations ensemble.

Or, si l'esprit prend corps dans la poésie au point qu'en elle, "l'audible, tout comme le visible, se trouve ravalé au rang de simple indice de l'Esprit "e que le rôle du matériau soit finalement nul, on est en droit de se demander en quoi la poésie est alors encore appelée un "art ". L'art ne se définit-il pas, dans le système de Hegel, comme un moment de réconciliation entre les deux opposés que sont le sensible et le spirituel ?' Est-il possible que la poésic soit penséc pure. ou presque? Sans doute est-ce le mépris de Hegel pour le sensible d'une part et son admiration sans borne pour la poésie lyrique l'autre part qui le conduisent à se contredire sur ce point, en accordant précisément une trop grande spiritualité à la forme poétique. Car si la poésie est déjà quasiment " prose de la pensée ", Hegel "a plus aucume justification pour dire que lesprit a besoin de la philosophie. pour trouver son expression adéquate. Ou alors, c'est que la réconciliation opérée par la philosophie est différente de celle opéréc par l'art, qưils ne médiatisent plus les mêmes contraires. En quoi aurait-on besoin d"une réconciliation conceptuelle si, dans la poésie. le sensible devient la voix même de l'esprit ?

Le caraclère sensible de la forme poétique est essentiel, et c’est parce qu’il le rend trop " dépourvu de valeur "que Hegel nous semble mettre quelque peu en péril sa dialectique dans ce passage. Le mol nest pas, comme le dit Hegel, insignifiant pour soi ; cest l'ordonnancement même des mots qui crée le sens, et ce contenu ne hui est pas exlérieur, ne lui préexiste pas séparément. La sonorité des mots, qui va pousser le poète à employer l'un plutôt que l'autre, est évidemment un élément constitutif majeur du texte. Si telle est bien sa pensée, il nous faut reconnaître que Hegel est bien réducteur (fuant à la nature de la poésie (prise au sens large qu’il cmploie) lorsqüil en fait presque un discours philosophique. Il en oublie la beauté, il oublie la jouissance des sonorités, et le fait que c’est précisément l'expression que l'on pourrait dire incroyable d'un "lond" de sens par ce véhicule formel fragile qui constitue l'intérêt même de la poésie épique, du théâtre ou, de façon plus évidente encore peut-être, de la poésie lyrique. Le plaisir esthétique, le goût que la poésie en général est apte à susciter viennent-ils uniquement de l'importance du contenu cognitif des phrases? La profondeur des thèmes soulevés, certes, contribue à en augmenter la valeur, mais il est évident que ce n'est pas seul ce qui en fait toute la valeur. Hegel en 
serait sans doute bien d'accord, si les conséquences de sa théorie ne le portaient pas à affirmer autre chose. Mais ce n'est pas là l'essentiel.

Il nous semble que ce passage l'enfonce dans un faux dilemme qu`il se crée lui-même. Ou bien, dans la poésie, le médium linguistique est ramené à un rang si minime, esl jugé si " insignifiant "(adjectif qu’il emploie). (que la poésie n"est plus vraiment un art, au sens où l'art aurait pour tâche de concilier le spirituel el le sensible. Et dans ce cas, on pourrait dire que c'est lart classique qui opère le mieux la réconciliation des deux. ear le fond y est exprimé adéquatement par la forme, landis que dans la poésic telle que nous la présente Hegel, et pis encore daus la philosophie. c'est le conceptuel qui domine, de sorte qu'on y trouve moins une "réconciliation" des deux éléments, le spirituel et le sensible, que l'écrasement de l'un par l'autre. Ou bien encore la poésie est bien un art., et même l'art le plus universel peut-être, mais alors il laut redonner ses lettres de noblesse au caractère sensible, matériel, qui est le sien - tâche peut-être bien impossible pour Hegel -- et dans ce cas, la poésie n'est plus nécessairement le moment où l'art "se transcende lui-même "au sens dialectique (c'est-à-dire au sens de créer un passage vers une sphère supérieure de l'expression du Concept), en ouvrant la voie à la philosophie. On peut, à la rigueur, suivre Hegel dans son cheminement dialectique el affirmer avec lui que l'art atteint sa perfection dans la poésie romantique; mais on peut le faire sans recourir à l'idée que cette perfection soit en même temps la marque de la fin du moment artistique, el de la nécessilé pour l'Esprit de s"incarner sous une autre forme. Sur quoi Hegel se fondet-il pour avancer' qu'il y a un aut-rlelà de l'art, si ce n'est sur le postulat métaphysique selon lequel la seule expression adćnuate de l'esprit est non sensible, postulat qui n'a de sens que dans son système el par lui ? Il y a ici une pétition de principe.

Ceci nous conduit à supposer que Ilegel emploie deux critères d'évaluation différents - et contradictoires - de la valeur hiérarchique d'une forme d'art sur l'autre: l'un esthétique, celui de l'adéquation du fond et de la forme, qui permet d'affirmer la supériorité de l'art classique et d'y situer la réalisation de la beauté parfaite ; el l'autre philosophique, ou métaphysique, qui voit le parachèvement de l'art dans une spiritualité plus grande. Or, de ce nouveau point de vue exclusivement philosophique, la beauté se trouve ramenée à un rang insignifiant, dans la mesure où les considérations sur le beau se trouvent subitement séparées des considérations sur le vrai auxquelles elles étaient jusqu'alors mêlées indistinctement, et qui de toute évidence seules comptent pour Hegel. Ceci impliquerait que l'apogée de la réconciliation Esprit/nature dans la forme artistique soit atteinte seulement au moment de la poésie, et non pas dans l'art classique. Et à l'inverse, si l'on suivait uniquement, de bout en bout, le critère esthétique de qualité, on pourrait envisager de clore la sphère artistique sur ses réalisations classiques. On briserait alors le 
passage que Hegel avait ménagé théoriquement vers une sphère supérieure de l'art (le moment de l'art romantique en général et celui de la poésie en particulier), de sorte que le processus dialectique serait interrompu.

De toute évidence, c'est donc le critère métaphysique, spirituel, qui régit l'évolution des formes d'art. Pourtant, Hegel annonçait au début de son Introduction que son ouvrage l'Esthétique serait "la science du beau ». En dépassant la perfection strictement esthétique de l'art classique pour subordonner à cette forme celle de l'art romantique en vertu de la plus grande détermination concrète du contenu spirituel des ceuves romantiques, Hegel semble bien faire un pas de trop vis-à-vis de son projet initial. Car ce passage de la forme classique à la forme romantique, ne se justifiant pas d'un point de vue esthétique, est tout simplement inutile vis-à-vis du type de réconciliation entre les deux opposés qui était demandé à l’art. C’est l'affirmation claire par IIegel qu'il ne fait pas la science du beau mais, une fois de plus, la science de l'esprit. Mais cela suppose aussi que la réconciliation de la philosophie est d'un autre type que celle de l'art. ce qui rend problématique sa supériorité si l'on maintient que les deux opposés sont bien les mêmes.

Nous avons tenté de comprendre les sources de la théorie hégélienne d'un dépassement de l'art par la philosophie dans l'entreprise de réconciliation du spirituel et du naturel. Toutefois. notre analyse nous a fait voir que s'il s'agit bien d'une tâche qui esı commune à l'art et à la philosophie, l'affirmation que la réconciliation philosophique est supérieure à celle de l'art pose problème. D’un côté, c'est vrai, nous avons vu que la nécessité de la médiation philosophique pouvait parfaitement se comprendre en vertu des postulats métaphysiques de base de Hegel, ce qui a été l'objet de notre première partie. D'un autre côté cependant, lorsqu'on entre dans le détail du moment esthétique. on trouve des fondements différents qui, menés à leur propre terme, conduisent à des conséquences contradictoires.

Le l'ait que nous nayons pas eu besoin de faire intervenir la notion d'homme dans notre analyse de la réconciliation opérée par lart - à la différence de la philosophie - indique déjà quassigner comme but commun à l'art, à la religion et à la philosophie, celui de "róconcilier " l’s opposés, revient finalement à assigner à chacun un but ùès différent. La seule justification possible pour expliquer gue la réconciliation opérée par la philosophie soit la plus parfaite serait que ce qu’elle médiatise soit plus élevé que ce que médiatise lart. Mais alors, cest bien de deux comples d'opposés différents qu il sagirait : dans un cas, le fond (spirituel) et la forme (sensible) : dans l'autre, l'esprit universel absolu et l'esprit particulier humain, aucun des couples ne pouvant, manifestement, être réduit à l'autre. Or, nous croyons que Hegel refuserait ce dualisme, car pour lui toutes les oppositions sont toujours issues d'une seule et même opposition 
fondamentale et constitutive, celle de l'esprit et de la nature, comme nous avons tenté de l'exposer sous ses diverses facettes dans la première partie de ce texte.

Il nous semble donc qu'il reste une contradiction, et que si ce sont bien les mêmes opposés qui sont réconciliés, il faut : ou bien garder le critère que nous avons appelé “ esthétique », el qui plaçait l'apogée de l'art dans la forme classique; ou bien utiliser le critère que nous avons appelé " métaphysique n et dire, comme le lail [Iegcel, que la plus haute spiritualisation de l'esprit se trouve dans la philosophie. Mais dans ce cas, la place de la poésie romantique vis-àvis de l'art classique devient problématique, du fait que Hegel affirmo toujours que c'est l'art classique qui. de toutes les formes d'art, opère le plus adéquatement la réconciliation en faisant que le fond se trouve parfaitement exprimé par la forme. Telles sont, tout du moins, les insuffisances et contradictions qui se révèlent à une lecture attentive au détail de l’argumentation même de Hegel. Ainsi, nous suggérons que toute la hiérarchie des arts repose sur des postulats qui sont utilisés alternativement par Hegel alor's qu'ils sont contradictoires, de sorte que sa notion de "réconciliation" s'en trouve double elle aussi.

Département de philosophie

Université d'Ottawa 